\title{
GASP: A Performance Analysis Tool Interface for Global Address Space Programming Models
}

\author{
Lawrence Berkeley National Lab Tech Report LBNL-61606 \\ Adam Leko $^{1}$, Dan Bonachea ${ }^{2}$, Hung-Hsun Su${ }^{1}$, Alan D. George ${ }^{1}$ \\ ${ }^{1}$ Electrical and Computer Engineering Dept., University of Florida \\ ${ }^{2}$ Computer Science Division, University of California at Berkeley
}

Specification Version 1.5, 09/14/2006

\section{Introduction}

\subsection{Scope}

Due to the wide range of compilers and the lack of a standardized performance tool interface, writers of performance tools face many challenges when incorporating support for global address space (GAS) programming models such as Unified Parallel C (UPC), Titanium, and Co-Array Fortran (CAF). This document presents a Global Address Space Performance tool interface (GASP) that is flexible enough to be adapted into current global address space compiler and runtime infrastructures with little effort, while allowing performance analysis tools to gather much information about the performance of global address space programs.

\subsection{Organization}

Section 2 gives a high-level overview of the GASP interface. As GASP can be used to support many global address space programming models, the interface has been broken down into model-independent and model-specific sections. Section 3 presents the model-independent portions of the GASP interface, and the subsequent sections detail the model-specific portions of the interface.

\subsection{Definitions}

- Model - a parallel programming language or library, such as UPC or MPI.

- Users - individuals using a GAS model such as UPC

- Developers - individuals who write parallel software infrastructure such as UPC, CAF, or Titanium compilers

- Tools - performance analysis tools such as Vampir, TAU, or KOJAK

- Tool developers - individuals who develop performance analysis tools

- Tool code - code or library implementing the tool developer's portion of the GASP interface

- Thread - a thread of control in a GAS program, maps directly to UPC's concept of threads or CAF's concept of images 


\section{GASP overview}

The GASP interface controls the interaction between a user's code, a performance tool, and GAS model compiler and/or runtime system. This interaction is event-based and comes in the form of callbacks to the gasp_event_notify function at runtime. The callbacks may come from instrumentation code placed directly in an executable, from an instrumented runtime library, or any other method; the interface only requires that gasp_event_notify is called at appropriate times in the manner described in the rest of this document.

The GASP interface allows tool developers to support GAS models on all platforms and implementations supporting the interface. The interface is used in the following three steps:

1. Users compile their GAS code using compiler wrapper scripts provided by tool developers. Users may specify which analysis they wish the tool to perform on their code through either command-line arguments, environment variables or through other tool-specific methods.

2. The compiler wrapper scripts pass appropriate flags to the compiler indicating which callbacks the tool wishes to receive. During the linking phase, the scripts link in appropriate code from the performance tool that handles the callbacks at runtime. This tool-provided code shall be written in C.

3. When a user runs their program, the tool-provided code receives callbacks at runtime and may perform some action such as storing all events in a trace file or performing basic statistical profiling.

The specifics of each step will be discussed in Section 3. The model-specific portions of the GASP interface will be discussed in the subsequent sections.

A GAS implementation may exclude any system-level event defined in the model-specific sections of this document if an application cannot be instrumented for that event (eg due to design limitations or other implementation-specific constraints).

Any action resulting in a violation of this specification shall result in undefined behavior. Tool and model implementors are strongly encouraged not to deviate from these specifications.

\section{Model-independent interface}

\subsection{Instrumentation control}

Instrumentation control is accomplished through either compilation arguments or compiler pragmas. Developers may use alternative names for the command-line arguments if the names specified below do not fit the conventions already used by the compiler.

\subsubsection{User-visible instrumentation control}

If a user wishes to instrument their code for use with a tool using the GASP interface, they shall pass one of the command-line arguments described in this section to the compiler wrapper scripts.

GASP system events are divided into the following broad categories, for the purposes of instrumentation control:

- Local access events: Events resulting from access to objects or variables contained in the portion of the global address space which is local to the accessing thread.

- User function events: Events resulting from entry and exit to user-defined functions, as described in section 4.3.1.

- Other events: Any system event which does not fall into the above categories.

The --inst argument specifies that the user's code shall be instrumented for all system events supported by the GAS model implementation which fall into the final category of events described above.

The --inst-local argument implies --inst, and additionally requests that user code shall be instrumented to generate local access events supported by the GAS model implementation. Otherwise, such events need not be generated.

For models lacking a semantic concept of local or remote memory accesses, --inst shall have the same semantics as --inst-local, implying instrumentation of all global address space accesses.

The--inst-functions argument implies --inst, and additionally requests that user code shall be instrumented to generate user function events supported by the GAS model implementation. Otherwise, such events need not be generated. 


\subsubsection{Tool-visible instrumentation control}

Compilers supporting the GASP interface shall provide the following command-line arguments for use by the tool-provided compiler wrapper scripts.

The arguments--inst, --inst-local and--inst-functions have the same semantics as the user-visible instrumentation flags specified in Section 3.1.1.

An additional argument--inst-only takes a single argument $f i l e n a m e$ which is a file containing a list of symbolic event names (as defined in the model-specific sections of this document) separated by newlines. The file's contents indicate the events for which the performance tool wishes to receive callbacks. Events in this file may be ignored by the compiler if the events are not supported by the model implementation. Compiler implementations are encouraged to avoid any overheads associated with generating events not specified by --inst-only, however tools that pass--inst-only must still be prepared to receive and ignore events which are not included in the --inst-only list.

\subsubsection{Interaction with instrumentation, measurement, and user events}

When code is compiled without an --inst flag, all instrumentation control shall be ignored and all user event callbacks shall be compiled away. Systems may link "dummy" versions of gasp_control and gasp_create_event (described in Sections 3.3 and 3.4) for applications that have no code compiled with --inst.

Systems may support compiling parts of an application using one of the --inst flags and compiling other parts of an application normally; for systems where this is not possible, this behavior may be prohibited. Applications compiled using an --inst flag for at least one translation unit shall also pass the --inst flag during the linking phase to the compiler wrapper scripts.

Any model-specific instrumentation control shall not have any effect on user events or on the state of measurement control. As a result, any model-specific instrumentation controls shall not prevent user events from being instrumented during compilation (e.g., \#pragma pupc shall not change the behavior of the pupc_create_event and pupc_event_start functions in UPC programs).

\subsection{Callback structure}

At runtime, all threads of an instrumented executable shall collectively call the gasp_init $\mathrm{C}$ function at the beginning of program execution after the model runtime has finished initialization but before executing the entry point in a user's code (e.g., main in UPC). The gasp_init function shall have the following signature:

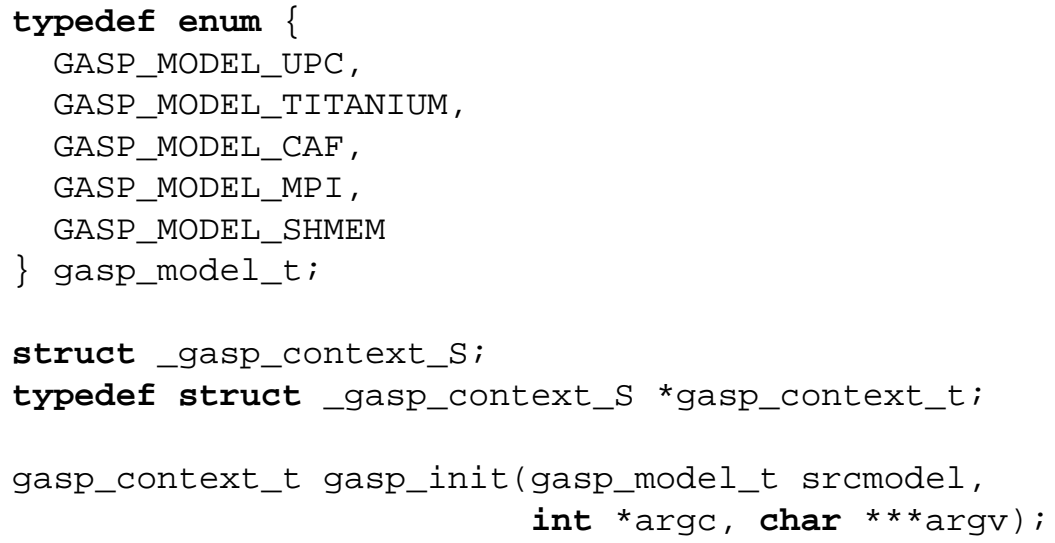

The gasp_init function and an implementation of the _gasp_context_S struct shall be provided by tool developers. A single running instance of an executable may collectively call gasp_init multiple times if the executable contains code written in multiple models (such as a hybrid UPC and CAF program), with at most one call per model.

The gasp_init function returns a pointer to an opaque, thread-specific, tool-implemented struct. This pointer shall be passed in all subsequent calls to the tool developer's code made on behalf of this thread. This pointer shall only be used in event callbacks for events corresponding to the model indicated by the srcmodel argument.

Tool code may modify the contents of the argc and argv pointers to support the processing of command-line arguments.

After the gasp_init function has been called by each thread of execution, the tool code shall receive all other callbacks through the two functions whose signatures are shown below: 


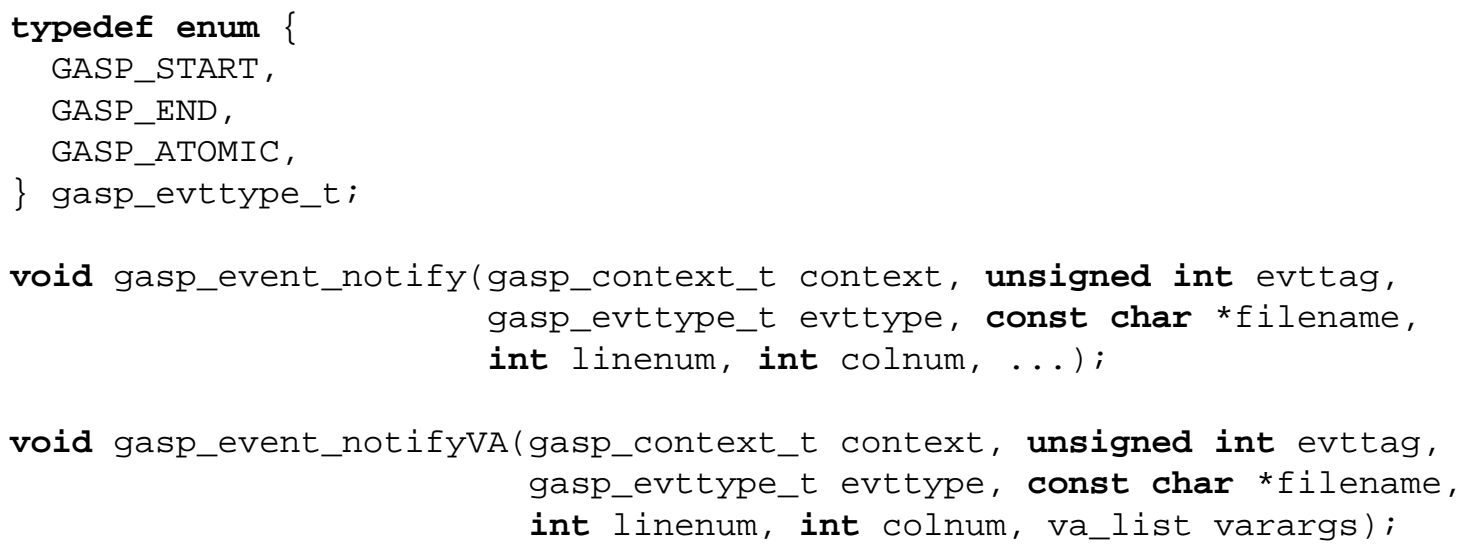

Both functions may be used interchangeably; the VA variant is provided as a convenience to developers.

The gasp_event_notify implementation shall be written in C, but may make upcalls to code written in the model specified by the srcmodel argument passed to the gasp_init function on the thread that received the callback. If upcalls are used, the gasp_event_notify function implementation is responsible for handling re-entrant calls. Additionally, code that is used in upcalls shall be compiled using the same environmental specifications as the code in a user's application (e.g., gasp_event_notify shall only perform upcalls to UPC code compiled under a static threads environment when used with a UPC program compiled under the static threads environment).

Any user data referenced by pointers passed to gasp_event_notify shall not be modified by tool code.

For the first argument to gasp_event_notify, tool code shall receive the same gasp_context_t pointer that was returned from the gasp_init function for this thread. Tool developers may use the context struct to store thread-local information for each thread. The gasp_event_notify function shall be thread-safe in order to support model implementations that make use of pthreads or other thread libraries.

The evttag argument shall specify the event identifier as described in the model-specific sections of this document. The evttype argument shall be of type gasp_evttype_t and shall indicate whether the event evttag is a begin event, end event, or atomic event.

The filename, linenum, and colnum arguments shall indicate the line and column number in the model-level source code most closely associated with the generation of the event evttag. If filename is non-NULL, it references a character string whose contents must remain valid and unmodified for the remainder of the program execution. The same filename pointer is permitted to be passed in multiple calls and by multiple threads, and it is also permitted for different filename pointers (passed in different calls) to indicate the same file name (this implies the tool may store filename pointer values and use simple pointer comparison of non-NULL values to establish filename equality, but not inequality).

GAS model implementations that do not retain column information during compilation may pass 0 in place of the colnum parameter. GAS model implementations that do not retain any source-level information during compilation may pass 0 for the filename, linenum, and colnum parameters. GAS model implementations are strongly encouraged to support these arguments unless this information can be efficiently and accurately obtained through other documented methods.

GAS model implementations that use instrumented runtime libraries for GASP support may provide dummy implementations for the gasp_event_notify, gasp_event_notifyVA,gasp_init functions and_gasp_context_S struct to prevent link errors while linking a user's application that is not being used with any performance tool.

The contents of the varargs argument shall be specific to each event identifier and type and will be discussed in the model-specific sections of this document.

\subsection{Measurement control}

Tool developers shall provide an implementation for the following function:

int gasp_control (gasp_context_t context, int on);

The gasp_control function takes the context argument in the same manner as the gasp_event_notify function.

When the value 0 is passed for the on parameter, the tool shall cease measuring any performance data associated with subsequent system or user events generated on the calling thread, until the thread makes a future call to gasp_control with a nonzero value for the on parameter. 
The gasp_control function shall return the last value for the on parameter the function received from this thread, or a nonzero value if gasp_control has never been called for this thread.

\subsection{User events}

Tool developers shall provide an implementation for the following function:

unsigned int gasp_create_event (gasp_context_t context,

const char *name, const char *desc);

The gasp_create_event shall return a tool-generated event identifier.

Compilers shall translate the corresponding model-specific_create_event functions listed in the model-specific sections of this document into corresponding gasp_create_event calls. The semantics of the name and desc arguments and the return value shall be the same as defined by the _create_event function listed in the model-specific section of this document corresponding to the model indicated by context.

\subsection{Header files}

Developers shall distribute a gasp.h $\mathrm{C}$ header file with their GAS model implementations that contains at least the following definitions:

- Function prototypes for the gasp_init, gasp_event_notify,gasp_control, and gasp_create_event functions and associated typedefs, enums, and structs.

- A GASP_VERSION macro that shall be defined to an integral date (coded as YYYYMMDD) corresponding to the GASP version supported by this GASP implementation. For implementations that support the version of GASP defined in this document, this macro shall be set to the integral value 20060914.

- Macro definitions that map the symbolic event names listed in the model-specific sections of this document to 32-bit unsigned integers.

The gasp. $\mathrm{h}$ file shall be installed in a directory that is included in the compiler's default search path.

\section{C interface}

\subsection{Instrumentation control}

Instrumentation for the events defined in this section shall be controlled by using the corresponding instrumentation control mechanisms for UPC code defined in Section 5.1.

\subsection{Measurement control}

Measurement for the events defined in this section shall be controlled by using the corresponding measurement control mechanisms for UPC code defined in Section 5.2.

\subsection{System events}

\subsubsection{Function events}

Table 1 shows system events related to executing user functions. 


\begin{tabular}{|c|c|c|}
\hline Symbo & Event type & vararg arguments \\
\hline GASP_C_FUN & Start, End & const char *funcsig \\
\hline
\end{tabular}

Table 1: User function events

These events occur upon each call to a user function (after entry into that function), and before exit from a user function (before returning to the caller as a result of executing a return statement or reaching the closing brace which terminates the function). The funcsig argument specifies the character string representing the full signature of the user function that is being entered or exited, or NULL if that information is not available.

If funcsig is non-NULL, it references a character string whose contents must remain valid and unmodified for the remainder of the program execution. The same funcsig pointer is permitted to be passed in multiple calls and by multiple threads, and it is also permitted for different funcsig pointers (passed in different calls) to indicate the same function signature (this implies the tool may store funcsig pointer values and use simple pointer comparison of non-NULL values to establish function equality, but not inequality).

\subsubsection{Memory allocation events}

Table 2 shows system events related to the standard memory allocation functions.

\begin{tabular}{|c|c|c|}
\hline Symbolic name & Event type & vararg arguments \\
\hline GASP_C_MALLOC & Start & size_t nbytes \\
\hline GASP_C_MALLOC & End & size_t nbytes, void *returnptr \\
\hline GASP_C_REALLOC & Start & void *ptr, size_t size \\
\hline GASP_C_REALLOC & End & void *ptr, size_t size, void *returnptr \\
\hline GASP_C_FREE & Start, End & void *ptr \\
\hline
\end{tabular}

Table 2: Memory allocation events

The GASP_C_MALLOC, GASP_C_REALLOC, and GASP_C_EREE stem directly from the standard C definitions of malloc, realloc, and free.

\subsection{Header files}

Supported C system events shall be handled in the same method as UPC events, which are described in Section 5.5.

\section{UPC interface}

\subsection{Instrumentation control}

Users may insert \#pragma pupc on or \#pragma pupc off directives in their code to instruct the compiler to avoid instrumenting lexically-scoped regions of a user's UPC code. These pragmas may be ignored by the compiler if the compiler cannot control instrumentation for arbitrary regions of code.

When an--inst argument is given to a compiler or compiler wrapper script, the \#pragma pupc shall default to on.

\subsection{Measurement control}

At runtime, users may call the following functions to control the measurement of performance data:

int pupc_control (int on);

The pupc_control function shall behave in the same manner as the gasp_control function defined in Section 3.3. 


\subsection{User events}

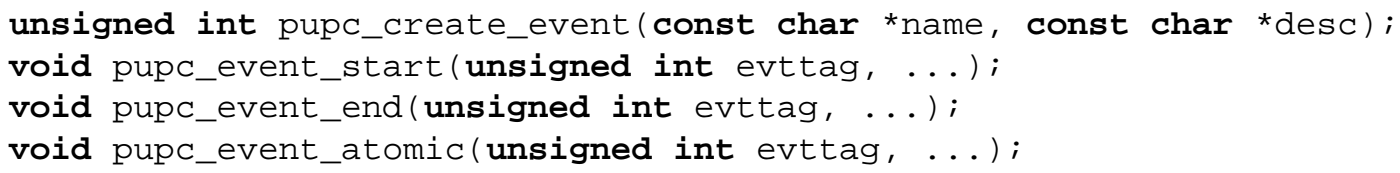

The pupc_create_event function shall be translated into a corresponding gasp_create_event call, as defined in Section 3.4. The name argument shall be used to associate a user-specified name with the event, and the desc argument may contain either NULL or a printf-style format string. The memory referenced by both arguments need not remain valid once the function returns.

The event identifier returned by pupc_create_event shall be a unique value in the range from GASP_UPC_USEREVT_START to GASP_UPC_USEREVT_END, inclusive. The GASP_UPC_USEREVT macros shall be provided in the gasp_upc. $\mathrm{h}$ header file described in Section 5.5. The value returned is thread-specific. If the unique identifiers are exhausted for the calling thread, pupc_create_event shall issue a fatal error.

The pupc_event_start,pupc_event_end, and pupc_event_atomic functions may be called by a user's UPC program at runtime. The evttag argument shall be any value returned by a prior pupc_create_event function call from the same thread. Users may pass in any list of values for the ... arguments, provided the argument types match the printf-style format string supplied in the corresponding pupc_create_event (according to the printf format string conventions specified by the target system). Any memory referenced by ... arguments (e.g. string arguments) need not remain valid once the function returns. A performance tool may use these values to display performance information alongside application-specific data captured during runtime to a user. The UPC implementation shall translate the pupc_event_start, pupc_event_end, and pupc_event_atomic function calls into corresponding gasp_event_notify function calls.

When a compiler does not receive any --inst arguments, the pupc_event function calls shall be excluded from the executable or linked against dummy implementations of these calls. A user's program shall not depend on any side effects that occur from executing the pupc_event functions.

Users shall not pass a shared-qualified pointer as an argument to the pupc_event functions.

\subsection{System events}

For the event arguments below, the UPC-specific types upc_flag_t and upc_op_t shall be converted to C ints.

Pointers to shared data shall be passed with an extra level of indirection, and may only be dereferenced through UPC upcalls. UPC implementations shall provide two opaque types, gasp_upc_PTS_t and gasp_upc_lock_t, which shall represent a generic pointer-to-shared (ie. shared void *), and a UPC lock pointer (ie. upc_lock_t $*$ ), respectively. These opaque types shall be typedef'ed to void to prevent $\mathrm{C}$ code from attempting to dereference them without using a cast in a UPC upcall. The content of any gasp_upc_PTS_t or gasp_upc_lock_t location passed to an event is only guaranteed to remain valid for the duration of the gasp_event_notify call, and must not be modified by the tool.

\subsubsection{Exit events}

Table 3 shows system events related to the end of a program's execution.

\begin{tabular}{|l|l|l|}
\hline Symbolic name & Event type & vararg arguments \\
\hline GASP_UPC_COLLECTIVE_EXIT & Start, End & int status \\
\hline GASP_UPC_NONCOLLECTIVE_EXIT & Atomic & int status \\
\hline
\end{tabular}

Table 3: Exit events

The GASP_UPC_COLLECTIVE_EXIT events shall occur at the end of a program's execution on each thread when a collective exit occurs. These events correspond to the execution of the final implicit barrier for UPC programs.

The GASP_UPC_NONCOLLECTIVE_EXIT event shall occur at the end of a program's execution on a single thread when a noncollective exit occurs.

\subsubsection{Synchronization events}

Table 4 shows events related to synchronization constructs. 


\begin{tabular}{|l|l|l|}
\hline Symbolic name & Event type & vararg arguments \\
\hline GASP_UPC_NOTIFY & Start, End & int named, int expr \\
\hline GASP_UPC_WAIT & Start, End & int named, int expr \\
\hline GASP_UPC_BARRIER & Start, End & int named, int expr \\
\hline GASP_UPC_FENCE & Start, End & (none) \\
\hline
\end{tabular}

Table 4: Synchronization events

These events shall occur before and after execution of the notify, wait, barrier, and fence synchronization statements. The named argument to the notify, wait, and barrier start events shall be nonzero if the user has provided an integer expression for the corresponding notify, wait, and barrier statements. In this case, the expr variable shall be set to the result of evaluating that integer expression. If the user has not provided an integer expression for the corresponding notify, wait, or barrier statements, the named argument shall be zero and the value of expr shall be undefined.

\subsubsection{Work-sharing events}

Table 5 shows events related to work-sharing constructs.

\begin{tabular}{|l|l|l|}
\hline Symbolic name & Event type & vararg arguments \\
\hline GASP_UPC_FORALL & Start, End & (none) \\
\hline
\end{tabular}

Table 5: Work-sharing events

These events shall occur on each thread before and after upc_forall constructs are executed.

\subsubsection{Library-related events}

Table 6 shows events related to library functions.

\begin{tabular}{|l|l|l|}
\hline Symbolic name & Event type & vararg arguments \\
\hline GASP_UPC_GLOBAL_ALLOC & Start & size_t nblocks, size_t nbytes \\
\hline GASP_UPC_GLOBAL_ALLOC & End & $\begin{array}{l}\text { size_t nblocks, size_t nbytes, } \\
\text { gasp_upc_PTS_t *newshrd_ptr }\end{array}$ \\
\hline GASP_UPC_ALL_ALLOC & Start & size_t nblocks, size_t nbytes \\
\hline GASP_UPC_ALL_ALLOC & End & $\begin{array}{l}\text { size_t nblocks, size_t nbytes, } \\
\text { gasp_upc_PTS_t *newshrd_ptr }\end{array}$ \\
\hline GASP_UPC_ALLOC & Start & size_t nbytes \\
\hline GASP_UPC_ALLOC & End & $\begin{array}{l}\text { size_t nbytes, } \\
\text { gasp_upc_PTS_t *newshrd_ptr }\end{array}$ \\
\hline GASP_UPC_FREE & Start, End & gasp_upc_PTS_t * shrd_ptr \\
\hline GASP_UPC_GLOBAL_LOCK_ALLOC & Start & (none) \\
\hline GASP_UPC_GLOBAL_LOCK_ALLOC & End & gasp_upc_lock_t *lck \\
\hline GASP_UPC_ALL_LOCK_ALLOC & Start & (none) \\
\hline GASP_UPC_ALL_LOCK_ALLOC & End & gasp_upc_lock_t *lck \\
\hline GASP_UPC_LOCK_FREE & Start, End & gasp_upc_lock_t *lck \\
\hline GASP_UPC_LOCK & Start, End & gasp_upc_lock_t *lck \\
\hline GASP_UPC_LOCK_ATTEMPT & Start & gasp_upc_lock_t *lck \\
\hline GASP_UPC_LOCK_ATTEMP T & End & $\begin{array}{l}\text { gasp_upc_lock_t * } \\
\text { int rek, }\end{array}$ \\
\hline GASP_UPC_UNLOCK & Start, End & gasp_upc_lock_t *lck \\
\hline & \multicolumn{2}{|l}{} \\
\hline
\end{tabular}




\begin{tabular}{|c|c|c|}
\hline Symbolic name & Event type & vararg arguments \\
\hline GASP_UPC_MEMCPY & Start, End & $\begin{array}{l}\text { gasp_upc_PTS_t }{ }^{*} \text { dst, } \\
\text { gasp_upc_PTS_t }{ }^{*} \text { src, } \\
\text { size_t } n\end{array}$ \\
\hline GASP_UPC_MEMGET & Start, End & $\begin{array}{l}\text { void *dst, } \\
\text { gasp_upc_PTS_t *src, } \\
\text { size_t n }\end{array}$ \\
\hline GASP_UPC_MEMPUT & Start, End & $\begin{array}{l}\text { gasp_upc_PTS_t *dst, } \\
\text { void *src, } \\
\text { size_t } n\end{array}$ \\
\hline GASP_UPC_MEMSET & Start, End & $\begin{array}{l}\text { gasp_upc_PTS_t *dst, } \\
\text { int } c, \\
\text { size_t } n\end{array}$ \\
\hline
\end{tabular}

Table 6: Library-related events

These events stem directly from the UPC library functions defined in the UPC specification. The vararg arguments for each event callback mirror those defined in the UPC language specification.

\subsubsection{Blocking shared variable access events}

Table 7 shows events related to blocking shared variable accesses.

\begin{tabular}{|l|l|l|}
\hline Symbolic name & Event type & vararg arguments \\
\hline GASP_UPC_GET & Start, End & $\begin{array}{l}\text { int is_relaxed, } \\
\text { void *dst, } \\
\text { gasp_upc_PTS_t *src, } \\
\text { size_t n }\end{array}$ \\
\hline GASP_UPC_PUT & Start, End & $\begin{array}{l}\text { int is_relaxed, } \\
\text { gasp_upc_PTS_t *dst, } \\
\text { void *src, } \\
\text { size_t n }\end{array}$ \\
\hline
\end{tabular}

Table 7: Blocking shared variable access events

These events shall occur whenever shared variables are assigned to or read from using the direct syntax (not using the upc.h library functions). The arguments to these events mimic those of the upc_memget and upc_memput event callback arguments, but differ from the ones presented in the previous section because they only arise from accessing shared variables directly. If the memory access occurs under the relaxed memory model, the is_relaxed parameter shall be nonzero; otherwise the is_relaxed parameter shall be zero.

\subsubsection{Nonblocking shared variable access events}

Table 8 shows events related to direct shared variable accesses implemented through nonblocking communication.

\begin{tabular}{|l|l|l|}
\hline Symbolic name & Event type & vararg arguments \\
\hline GASP_UPC_NB_GET_INIT & Start & $\begin{array}{l}\text { int is_relaxed, } \\
\text { void *dst, } \\
\text { gasp_upc_PTS_t * } \\
\text { size_t n }\end{array}$ \\
\hline \multicolumn{2}{|c|}{ Continued on next page } \\
\hline
\end{tabular}




\begin{tabular}{|c|c|c|}
\hline Symbolic name & Event type & vararg arguments \\
\hline GASP_UPC_NB_GET_INIT & End & $\begin{array}{l}\text { int is_relaxed, } \\
\text { void *dst, } \\
\text { gasp_upc_PTS_t *src, } \\
\text { size_t n, } \\
\text { gasp_upc_nb_handle_t handle }\end{array}$ \\
\hline GASP_UPC_NB_GET_DATA & Start, End & gasp_upc_nb_handle_t handle \\
\hline GASP_UPC_NB_PUT_INIT & Start & $\begin{array}{l}\text { int is_relaxed, } \\
\text { gasp_upc_PTS_t *dst, } \\
\text { void *src, } \\
\text { size_t } n\end{array}$ \\
\hline GASP_UPC_NB_PUT_INIT & End & $\begin{array}{l}\text { int is_relaxed, } \\
\text { gasp_upc_PTS_t *dst, } \\
\text { void *src, } \\
\text { size_t n, } \\
\text { gasp_upc_nb_handle_t handle }\end{array}$ \\
\hline GASP_UPC_NB_PUT_DATA & Start, End & gasp_upc_nb_handle_t handle \\
\hline GASP_UPC_NB_SYNC & Start, End & gasp_upc_nb_handle_t handle \\
\hline
\end{tabular}

Table 8: Nonblocking shared variable access events

These nonblocking direct shared variable access events are similar to the regular direct shared variable access events in Section 5.4.5. The INIT events shall correspond to the nonblocking communication initiation, the DATA events shall correspond to when the data starts to arrive and completely arrives on the destination node (these events may be excluded for most implementations that use hardware-supported DMA), and the GASP_UPC_NB_SYNC function shall correspond to the final synchronization call that blocks until the corresponding data of the nonblocking operation is no longer in flight.

gasp_upc_nb_handle_t shall be an opaque type defined by the UPC implementation. Several outstanding nonblocking get or put operations may be attached to a single gasp_upc_nb_handle_t instance. When a sync callback is received, the tool code shall assume all get and put operations for the corresponding handle in the sync callback have been retired.

The implementation may pass the handle GASP_NB_TRIVIAL to GASP_UPC_NB_\{PUT, GET \}_INIT to indicate the operation was completed synchronously in the initiation interval. The tool should ignore any DATA or SYNC event callbacks with the handle GASP_NB_TRIVIAL.

\subsubsection{Shared variable cache events}

Table 9 shows events related to shared variable cache events.

\begin{tabular}{|l|l|l|}
\hline Symbolic name & Event type & vararg arguments \\
\hline GASP_UPC_CACHE_MISS & Atomic & $\begin{array}{l}\text { size_t n, } \\
\text { size_t n_lines }\end{array}$ \\
\hline GASP_UPC_CACHE_HIT & Atomic & size_t n \\
\hline GASP_UPC_CACHE_INVALIDATE & Atomic & size_t n_dirty \\
\hline
\end{tabular}

Table 9: Shared variable cache events

The GASP_UPC_CACHE events may be sent for UPC runtime systems containing a software cache after a corresponding get or put start event but before a corresponding get or put end event (including nonblocking communication events). UPC runtimes using write-through cache systems may send GASP_UPC_CACHE_MISS events for each corresponding put event.

The size_t $n$ argument for the MISS and HIT events shall indicate the amount of data read from the cache line for the particular cache hit or cache miss.

The n_lines argument of the GASP_UPC_CACHE_MISS event shall indicate the number of bytes brought into the cache as a result of the miss (in most cases, the line size of the cache). 
The n_dirty argument of the GASP_UPC_CACHE_INVALIDATE shall indicate the number of dirty cache lines that were written back to shared memory due to a cache line invalidation.

\subsubsection{Collective communication events}

Table 10 shows events related to collective communication.

\begin{tabular}{|c|c|c|}
\hline Symbolic name & Event type & vararg arguments \\
\hline GASP_UPC_ALL_BROADCAST & Start, End & $\begin{array}{l}\text { gasp_upc_PTS_t *dst, } \\
\text { gasp_upc_PTS_t *src, } \\
\text { size_t nbytes, } \\
\text { int upc_flags }\end{array}$ \\
\hline GASP_UPC_ALL_SCATTER & Start, End & $\begin{array}{l}\text { gasp_upc_PTS_t *dst, } \\
\text { gasp_upc_PTS_t *src, } \\
\text { size_t nbytes, } \\
\text { int upc_flags }\end{array}$ \\
\hline GASP_UPC_ALL_GATHER & Start, End & $\begin{array}{l}\text { gasp_upc_PTS_t *dst, } \\
\text { gasp_upc_PTS_t *src, } \\
\text { size_t nbytes, } \\
\text { int upc_flags }\end{array}$ \\
\hline GASP_UPC_ALL_GATHER_ALL & Start, End & $\begin{array}{l}\text { gasp_upc_PTS_t *dst, } \\
\text { gasp_upc_PTS_t }{ }^{\text {src, }} \\
\text { size_t nbytes, } \\
\text { int upc_flags }\end{array}$ \\
\hline GASP_UPC_ALL_EXCHANGE & Start, End & $\begin{array}{l}\text { gasp_upc_PTS_t *dst, } \\
\text { gasp_upc_PTS_t }{ }^{*} \text { src, } \\
\text { size_t nbytes, } \\
\text { int upc_flags }\end{array}$ \\
\hline GASP_UPC_ALL_PERMUTE & Start, End & $\begin{array}{l}\text { gasp_upc_PTS_t *dst, } \\
\text { gasp_upc_PTS_t *src, } \\
\text { gasp_upc_PTS_t *perm, } \\
\text { size_t nbytes, } \\
\text { int upc_flags }\end{array}$ \\
\hline GASP_UPC_ALL_REDUCE & Start, End & $\begin{array}{l}\text { gasp_upc_PTS_t *dst, } \\
\text { gasp_upc_PTS_t *src, } \\
\text { int upc_op, } \\
\text { size_t nelems, } \\
\text { size_t blk_size, } \\
\text { void } \star f u n c, \\
\text { int upc_flags, } \\
\text { gasp_upc_reduction_t type }\end{array}$ \\
\hline GASP_UPC_ALL_PREFIX_REDUCE & Start, End & $\begin{array}{l}\text { gasp_upc_PTS_t *dst, } \\
\text { gasp_upc_PTS_t *src, } \\
\text { int upc_op, } \\
\text { size_t nelems, } \\
\text { size_t blk_size, } \\
\text { void *func, } \\
\text { int upc_flags, } \\
\text { gasp_upc_reduction_t type }\end{array}$ \\
\hline
\end{tabular}

Table 10: Collective communication events

The events in Table 10 stem directly from the UPC collective library functions defined in the UPC specification. The vararg arguments for each event callback mirror those defined in the UPC language specification.

For the reduction functions, the gasp_upc_reduction_t enum shall be provided by a UPC implementation and shall be defined as follows: 


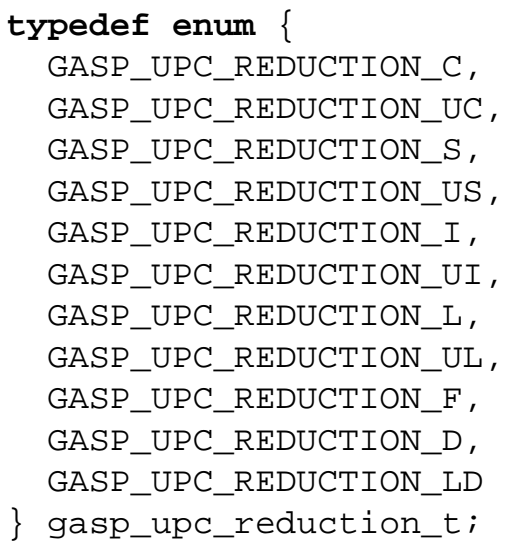

where the suffix to GASP_UPC_REDUCTION denotes the same type as specified in the UPC specification.

\subsection{Header files}

UPC compilers shall distribute a pupc . h $\mathrm{C}$ header file with their GAS language implementations that contains function prototypes for the functions defined in Sections 5.2 and 5.3. The pupc.h file shall be installed in a directory that is included in the UPC compiler's default search path.

All supported system events and associated gasp_upc_* types shall be defined in a gasp_upc.h file located in the same directory as the gasp.h file. System events not supported by an implementation shall not be included in the gasp_upc.h file. The gasp_upc. $\mathrm{h}$ header file may include definitions for implementation-specific events, along with brief documentation embedded in source code comments.

Compilers shall define a compiler-specific integral GASP_UPC_VERSION version number in gasp_upc.h that may be incremented when new implementation-specific events are added. Compiler developers are encouraged to use the GASP_X_Y naming convention for all implementation-specific events, where $\mathrm{X}$ is an abbreviation for their compilation system (such as BUPC) and $\mathrm{Y}$ is a short, descriptive name for each event.

Compilers that implement the pupc interface shall predefine the feature macro__ UPC_PUPC_ to the value 1 . The macro should be predefined whenever applications may safely \#include <pupc.h>, invoke the functions it defines and use the \#pragma pupc directives, without causing any translation errors. The feature macro does not guarantee that GASP instrumentation is actually enabled for a given compilation, as some of the features might have no effect in non-instrumenting compilations.

\section{Other models}

Event sets for additional models (SHMEM, Titanium, CAF, MPI, etc.) will be defined in a future revision of this specification. 